\title{
Artigo/Article
}

\section{Caracterização da tuberculose multidroga resistente durante a gravidez, em Campinas, Estado de São Paulo, Brasil, no período de 1995 a 2007}

\author{
Characterization of multidrug-resistant tuberculosis during pregnancy in Campinas, \\ State of São Paulo, Brazil, from 1995 to 2007
}

Helenice Bosco de Oliveira ${ }^{1}$ e Silvia Helena Rondina Mateus ${ }^{2}$

\begin{abstract}
RESUMO
Introdução: Foi feito um acompanhamento das gestantes tratadas para tuberculose multidroga resistente (TB-MDR) durante a gravidez, em Campinas, Estado de São Paulo, no período de 1995 a 2007. Métodos: No estudo retrospectivo, foram incluídas pacientes com cepa resistente, pelo menos à isoniazida e à rifampicina e gravidez em qualquer momento, durante o tratamento. Os casos receberam esquema individualizado baseado nos padrões de resistência medicamentosa e nos antecedentes de tratamento de cada paciente. Resultados: Sete gestantes apresentaram resistência a duas ou mais drogas. Três já estavam grávidas, antes do início do tratamento e quatro engravidaram depois. Duas estavam com Aids, uma foi a óbito e em outra ocorreu falência de tratamento. Todas apresentaram tuberculose avançada com achados radiológicos graves e para cinco pacientes o tempo médio da conversão do escarro foi de quatro meses. Duas gestantes foram curadas. Dois recém-nascidos foram infectados com o HIV por suas progenitoras. Conclusões: $\mathrm{O}$ resultado do tratamento foi insatisfatório e a supervisão direta é imperativa na TB-MDR durante a gestação. O planejamento familiar deve ser fortemente recomendado.
\end{abstract}

Palavras-chaves: Tuberculose. Multidroga resistente. Gravidez.

\begin{abstract}
Introduction: There was a follow-up of pregnant women treated for multidrug-resistance tuberculosis (MDR-TB) during pregnancy in Campinas, State of São Paulo, Brazil, from 1995 to 2007. Methods: In a retrospective study, patients with tuberculosis who were resistant to at least isoniazid and rifampicin and had pregnancy at any time during the treatment were included. The cases were individually treated, considering drug susceptibility test results and patients' prior treatments. Results: Seven cases presented resistance to two or more drugs. Three were already pregnant before the beginning of the treatment, and four conceived after. Two were with AIDS; one died, and the therapeutic program in the other one failed. All showed advanced tuberculosis with acute radiological findings, and the median sputum conversion time for five patients was four months. Two patients were cured. Two newborn babies were infected with HIV by their mothers. Conclusions: The outcome was unsatisfactory, and direct supervision is imperative in MDR-TB during pregnancy. The family planning program should be strongly recommended.
\end{abstract}

Keywords: Tuberculosis. Multidrug resistance. Pregnancy.

1. Departamento de Medicina Preventiva e Social, Universidade Estadual de Campinas, Campinas, SP. 2. Policlínica II, Prefeitura Municipal de Campinas, Campinas, SP.

Endereço para correspondência: Dra. Helenice Bosco de Oliveira. Rua Waldyr Aparecido da Silva 60, Barão Geraldo, 13085-065 Campinas, SP.

Tel: 55 19 3289-4009; Fax: 55 193521-8036

e-mail: helenice@unicamp.br

Recebido para publicação em 17/08/2010

Aceito em 01/06/2011

\section{INTRODUÇÃO}

A tuberculose (TB) na gravidez pode ser tratada com sucesso, utilizando drogas não teratogênicas como isoniazida, rifampicina e pirazinamida; entretanto, a doença não curada tem sido associada à morbimortalidade, entre mulheres grávidas, com possibilidade de disseminação pela veia umbelical, pela transmissão pós-parto para o neonato e outros contactuantes $^{1}$.

A resistência pelo menos à rifampicina e à isoniazida na gestação é rara e o tratamento para tuberculose multidroga resistente (TB-MDR), durante a gravidez, ainda é controverso. São escassos os trabalhos publicados e pequeno o número de pacientes nestes estudos ${ }^{2-9}$. Também, pouco se conhece sobre a segurança dos medicamentos para o tratamento da TB-MDR durante a gestação: alguns são contraindicados; os esquemas são mais agressivos, menos efetivos, evitados no primeiro trimestre e com reduzida probabilidade de cura. Alguns autores recomendam o uso dos medicamentos após a gravidez ${ }^{3}$. No entanto, os clínicos devem avaliar o risco de disseminação para a mãe e para a criança e os efeitos adversos pela administração das drogas para TB-MDR.

Diante de uma situação especial, em mulheres grávidas, e também pelas dificuldades no manejo dos esquemas terapêuticos, este estudo objetivou conhecer as características destas pacientes com TB-MDR em relação aos antecedentes do uso de tuberculostáticos, perfil de resistências, presença de reações adversas e desfecho do tratamento e da gestação.

\section{MÉTODOS}

Foi pesquisada uma coorte retrospectiva de pacientes com TB-MDR secundária tratados em ambulatório público de referência na Cidade de Campinas-SP, no período de 13 anos, de 1995 a 
2007, e que recebe doentes vindos de cidades da região e até de outros estados. Os pacientes, quando encaminhados, já foram tratados em suas cidades de origem com esquemas de primeira linha e de segunda linha para falência de terapêutica.

Os critérios de inclusão neste estudo foram: I - casos com cepa resistente a pelo menos isoniazida e rifampicina; e II - gravidez em qualquer momento durante o tratamento.

As pacientes receberam esquemas individualizados baseado nos padrões de resistência medicamentosa, nos antecedentes de tratamento de cada paciente e no esquema preconizado pelo Ministério da Saúde (amicacina, ofloxacina, estreptomicina, terizidona/ciclocerina, etambutol, pirazinamida). Amicacina e estreptomicina foram utilizadas, apenas, previamente à detecção da gravidez.

\section{Considerações éticas}

O estudo foi aprovado pelo Comitê de Ética e Pesquisa da Faculdade de Ciências Médicas da Universidade Estadual de Campinas-UNICAMP.

\section{RESULTADOS}

Sete casos de TB-MDR secundária foram identificados durante a gravidez, todos infectados com cepas de M. tuberculosis: um deles com bacilo resistente a duas drogas, três pacientes a três drogas, outras três a quatro drogas e o tempo médio de doença foi de 4,6 anos (mediana de 4 anos) até o início do esquema para resistência (Tabela 1 ).
Duas pacientes tinham Aids (Tabela 1) e todas apresentavam TB avançada com achados radiológicos graves. A idade média no início da gestação foi de 25,4 anos (mediana de 25 anos) (Tabela 1). Três mulheres já estavam grávidas antes do início do esquema para TB-MDR; quatro engravidaram durante o tratamento (com 21 semanas, no $11^{\circ}$ mês, no $12^{\circ}$ e no $15^{\circ}$ ).

Para cinco pacientes o tempo médio da conversão da cultura do escarro foi de quatro meses (variação 2-10) (Tabela 1). Quatro mulheres apresentaram baciloscopia positiva no parto. Não se observou nenhum efeito adverso sério durante a gravidez.

Foi verificado que três pacientes foram a óbito, ocorreu falência do esquema terapêutico em uma gestante e duas transferências. Em setembro de 2009, constatou-se que a paciente 6 foi transferida para hospital especializado e só foi possível obter a informação de que estava internada em dezembro de 2007; a paciente 2, com resistência à isoniazida e à rifampicina, foi transferida para unidade básica de outro município, tendo sido curada e sem apresentar recidiva até o momento. Uma outra paciente também se curou. Todas receberam cuidados de pré-natal e para uma o parto foi prematuro. Não ocorreu nenhum óbito durante a gravidez.

Ao nascimento, não se observaram complicações congênitas ou neonatais. Dois recém-nascidos ( $\mathrm{RN}$ ) com exposição ao HIV foram medicados com zidovudine e permaneceram assintomáticos durante o seguimento (Tabela 2). Duas crianças foram amamentadas, sendo que para uma delas a mãe utilizou máscara N95. Seis neonatos foram

TABELA 1 - Características epidemiológicas das gestantes em tratamento para tuberculose multidroga resistente em Campinas-SP, de 1995 a 2007.

\begin{tabular}{|c|c|c|c|c|c|c|c|c|c|}
\hline Paciente & $\begin{array}{c}\text { Duração da } \\
\text { TB até o } \\
\text { esquema } \\
\text { de MDR }\end{array}$ & $\begin{array}{l}\text { Perfil de } \\
\text { resistência } \\
\text { prévio ao } \\
\text { esquema } \\
\text { de MDR }\end{array}$ & $\begin{array}{c}\text { Resultado } \\
\text { do teste } \\
\text { HIV }\end{array}$ & $\begin{array}{l}\text { Idade na } \\
\text { gestação }\end{array}$ & $\begin{array}{c}\text { Gravidez } \\
\text { quando } \\
\text { iniciou } \\
\text { tratamento }\end{array}$ & $\begin{array}{l}\text { Época do } \\
\text { tratamento } \\
\text { em que } \\
\text { engravidou }\end{array}$ & $\begin{array}{c}\text { Mês de } \\
\text { conversão } \\
\text { da cultura }\end{array}$ & $\begin{array}{c}\text { Baciloscopia de } \\
\text { escarro no } \\
\text { parto }\end{array}$ & $\begin{array}{c}\text { Resultado do tratamento } \\
\text { materno }\end{array}$ \\
\hline 1 & 7 anos & R, I, Z, S & negativo & 18 & não & $12 \mathrm{~m}$ & não & positivo & óbito \\
\hline 3 & 8 anos & $\mathrm{R}, \mathrm{I}, \mathrm{Z}, \mathrm{M}$ & negativo & 28 & não & $21 \mathrm{~s}$ & $10^{\circ}$ & positivo & óbito \\
\hline 4 & 4 anos & $\mathrm{R}, \mathrm{I}, \mathrm{Z}$ & negativo & 25 & não & $15 \mathrm{~m}$ & $3^{\circ}$ & negativo & cura \\
\hline 5 & 4 anos & $\mathrm{R}, \mathrm{I}, \mathrm{M}$ & positivo & 36 & não & $11 \mathrm{~m}$ & $3^{\circ}$ & negativo & óbito \\
\hline 6 & 1 ano & $\mathrm{R}, \mathrm{I}, \mathrm{Z}, \mathrm{S}$ & negativo & 23 & $\operatorname{sim}, 9 s$ & - & não & positivo & transferência seguida de cura \\
\hline 7 & 3 anos & I, M, S & positivo & 26 & $\operatorname{sim}, 27 \mathrm{~s}$ & - & $2^{\circ}$ & negativo & falência \\
\hline
\end{tabular}

TB: tuberculose, MDR: multidroga resistente. R: rifampicina, I: isoniazida, Z: pyrazinamida, S: estreptomicina, M: etambutol, s: semanas, m: mês. HIV: vírus da imunodeficiência adquirida.

TABELA 2 - Características dos neonatos relacionadas ao tratamento de tuberculose multidroga resistente durante a gravidez em Campinas-SP, de 1995 a 2007.

\begin{tabular}{|c|c|c|c|c|c|c|c|}
\hline $\begin{array}{l}\text { Recém } \\
\text { nascido }\end{array}$ & $\begin{array}{l}\text { Tempo de exposição ao } \\
\text { esquema de TB-MDR }\end{array}$ & $\begin{array}{l}\text { Condições ao } \\
\text { nascimento }\end{array}$ & Quimioprofilaxia & BCG & Amamentação & $\begin{array}{c}\text { Tempo de } \\
\text { acompanhamento }\end{array}$ & Adoecimento \\
\hline$\overline{1}$ & $9 \mathrm{~m}$ & sadio & sem informação & sem informação & sem informação & $18 \mathrm{~m}$ & não \\
\hline 3 & $5 \mathrm{~m}$ & sadio & $\operatorname{sim}$ & sim, após quimio & amamentou com máscara & 3 anos & não \\
\hline 4 & $9 m$ & sadio & não & sim, ao nascer & $\operatorname{sim}$ & 6 anos & não \\
\hline \multirow[t]{2}{*}{5} & $8 \mathrm{~m}$ & com HIV+ & & & & & \\
\hline & & uso de AZT & não & sim, ao nascer & não & 3 anos & não \\
\hline \multirow[t]{2}{*}{6} & $5 \mathrm{~m}$ & prematuro com & não & sim, após alta da & não & $28 \mathrm{~m}$ & não \\
\hline & & $30 \mathrm{~s}$ & & UTI neonatal & & & \\
\hline \multirow[t]{2}{*}{7} & $2 \mathrm{~m}$ & com HIV+ & & & & & \\
\hline & & uso de AZT & não & sim, ao nascer & não & $12 \mathrm{~m}$ & Não \\
\hline
\end{tabular}

TB-MDR: tuberculose multidroga resistente, BCG: Vacinação BCG, m: mês, s: semanas, HIV+: (vírus da imunodeficiência adquirida), AZT: zidovudina, UTI: unidade de tratamento intensivo. 
vacinados com BCG intradérmico (Tabela 2). Não foi possível obter informação sobre presença de lesões em placenta para investigar a possibilidade de transmissão vertical, nem da realização de culturas de lavado gástrico.

As crianças não foram acompanhadas pelo ambulatório de referência e durante o tratamento das mães não houve relato de adoecimento ou de quadro sugestivo de TB.

\section{DISCUSSÃO}

Estudos sobre TB-MDR na gravidez estão limitados a $64 \operatorname{casos}^{2-9}$ O enfoque dado pelos autores é essencialmente clínico e apenas em Palácios cols ${ }^{8}$, no Peru, em uma coorte de 10 anos, as 38 pacientes foram caracterizadas em relação ao desfecho do tratamento e da gestação.

As gestantes em nosso trabalho fazem parte de um grupo de doentes, homens e mulheres, encaminhados durante 13 anos para tratamento em ambulatório de referência após serem esgotadas as alternativas para tratamento autoadministrado em unidades básicas. Não fazem parte de uma coorte subjacente de mulheres grávidas, vieram de cidades vizinhas e até de outros estados. A constatação de 4,6 anos como tempo médio de doença, antes do esquema para TB$\mathrm{MDR}$, evidencia que o processo da resistência na TB é grave para os doentes que foram tratados previamente, sem sucesso. Nesse período, devem ter ocorrido reinícios de tratamento, abandonos repetidos, recidivas, irregularidades na ingestão das drogas, agravando a doença ${ }^{10}$.

$\mathrm{O}$ resultado do tratamento materno foi insatisfatório, mesmo com a regularidade ao esquema MDR pelas gestantes e não se pode apontar negligência pelo serviço. A ocorrência de óbitos se deu meses após o parto, sendo que em uma paciente, aparentemente bem, apresentou hemoptise incontrolável e em outras duas o óbito ocorreu pela deterioração do quadro pulmonar e insuficiência respiratória, e uma delas apresentava Aids. A paciente 7, também com Aids, teve falência como resultado para o esquema de TB-MDR e até 2007 , quando ocorreu a falência, não havia no ambulatório de referência disponibilidade de linesolide ou derivados de anasamicina (rifabutina, rifapentina). A estratégia directly observed therapy short-course (DOTS) ainda não era preconizada em 1995 e quando implantada foi bastante irregular e, para as gestantes, que vieram de outros municípios, esta ação não foi realizada. Por outro lado, um estudo peruano ${ }^{8}$ mostrou $58,1 \%$ de cura entre gestantes TB-MDR confirmada, que foi atribuída ao suporte por agentes comunitários da ONG Sócios em Salud para DOTS domiciliar e também de auxílios socioeconômico e psicossocial. Na Cidade de Campinas, em 2009, o resultado do tratamento para casos novos obteve cura em 75,9\%, óbitos por todas as causas em 8,3\% (re-tratamentos 61\% e 5,1\%, respectivamente). O Sistema de Vigilância Epidemiológica da TBMDR, em 2008, registrou cura de 50\% e óbito em 12,5\%. Portanto, isto sugere que no grupo de gestantes haveria necessidade de se pensar em ações de saúde pública interativas envolvendo clínica, suporte socioeconômico e psicológico para auxiliar na melhoria da saúde deste grupo de pacientes.

Holtz cols ${ }^{11}$ referem como preditores para o sucesso do resultado final do tratamento, o número de drogas usadas, o alto índice de massa corporal e a sensibilidade à ofloxacina pelo efeito bactericida e esterilizante das fluorquinolonas. Também, pacientes com conversão mais precoce da cultura de escarro tiveram mais sucesso no tratamento.
Apesar das limitações desta investigação retrospectiva, com a utilização de dados secundários, não foram observadas reações colaterais, mesmo na associação com o HIV em duas gestantes, diferente do verificado por outros autores ${ }^{3}$ que relataram hepatite e surdez como complicações relacionadas às drogas. Em LimaPerú ${ }^{12}$, foi constatado que, embora os efeitos adversos tenham sido frequentes, não ocorreu descontinuidade do tratamento e em apenas $11,7 \%$ resultou na suspensão de um dos medicamentos.

Inúmeros dilemas permearam o tratamento das pacientes neste estudo: em primeiro lugar, o tratamento da TB-MDR na gestação ainda é polêmico, com exposição intraútero a drogas com potencial teratogênico e que incluem efeitos ototóxicos, neurotóxicos e nas cartilagens. Experiências em maior escala com fármacos para TB-MDR ainda não estão disponíveis e alguns autores recomendam que o uso de drogas para TB-MDR seja postergado para o segundo trimestre da gravidez, exceto em gestantes com doença clinicamente avançada ${ }^{3}$. Outros autores consideram que deve ser dada a opção de continuar o tratamento para MDR ao invés de descontinuar o esquema ou mesmo interromper a gravidez ${ }^{2}$. Nesta série de casos, não foram diagnosticadas anomalias ou tuberculose congênita, semelhante a outros estudos citados ${ }^{2-5}$. Por outro lado, o tratamento com esquema terapêutico cuidadosamente selecionado, segundo perfil de resistência e trimestre da gravidez, poderá proteger a mãe da progressão da doença e de um risco obstétrico maior, da prematuridade, do baixo peso ao nascer, bem como da transmissão vertical e da transmissão pós-parto ${ }^{2}$.

Em segundo lugar, a chance de transmissão para o recémnascido não pode ser subestimada. Na comparação, entre risco de infecção entre contatos de pacientes sem tratamento prévio com doentes com tratamento anterior, sensíveis ou resistentes, não foi evidenciada menor infectividade entre os resistentes ${ }^{13}$ e a ocorrência de tuberculose-doença entre contatos intradomiciliares de TB-MDR e TB sensível foi semelhante. O maior risco de infecção entre contactos de doentes resistentes seria explicado pela exposição mais prolongada do que pelo aumento da infecciosidade do bacilo resistente. Por outro lado, o fato do esquema para tratamento da TBMDRser menos eficaz mantém o paciente infeccioso por mais tempo comparado àqueles com micobactéria sensível. Foi verificado ${ }^{11}$ que, em condições de programa de tratamento, a maioria dos pacientes com TB-MDR apresentou conversão da cultura de escarro até 12 semanas do início do esquema; neste estudo, a negativação se deu em média com quatro meses, permitindo maior chance de exposição aos bacilos resistentes.

Para os RN, a indicação de quimioprofilaxia não é conclusiva e a Organização Mundial de Saúde não recomenda o uso universal de drogas de segunda linha para quimioprofilaxia em contatos de TB-MDR. Estes contatos com diagnóstico de infecção latente não estarão necessariamente infectados com a mesma cepa do doente resistente. $\mathrm{O}$ teste tuberculínico pode se tornar reator em pacientes com TB independente da cepa ser sensível ou resistente. No entanto, crianças contactuantes íntimos de pacientes com TB-MDR e que desenvolvem a doença geralmente apresentam resistência primária transmitida pelo caso índice com resistência medicamentosa ${ }^{14}$. Uma quimioprofilaxia apropriada, levando em consideração o perfil de resistência do caso índice, parece ser mais efetiva em prevenir o desenvolvimento da doença ${ }^{14}$. Na literatura, é encontrada, ainda, a indicação de separar a criança da mãe em tratamento para TBMDR pela prolongada infectividade, ausência de esquema eficaz de 
quimioprofilaxia e quando há falta de adesão da mãe ao tratamento ${ }^{15}$. Neste estudo, um $\mathrm{RN}$ recebeu isoniazida por seis meses sendo vacinado em seguida e outros cinco foram vacinados com BCG logo após o nascimento, não havendo relato de adoecimento até a data final da coleta dos dados; as crianças permaneceram com as mães.

Em relação à amamentação, apenas dois $\mathrm{RN}$ foram aleitados. Os antituberculostáticos podem ser encontrados no leite materno em concentrações menores que a dose terapêutica usada para crianças. Entretanto, os dados sobre os efeitos desta exposição durante o tratamento da TB-MDR são limitados.

Deve ainda ser lembrado que embora a mãe permaneça com baciloscopia negativa, a presença de quadro sugestivo de TB, ou mesmo resultado de teste tuberculínico reator e achado radiológico durante o acompanhamento da criança implica em continuar a investigação de contactuantes: outros pacientes com TB-MDR podem ser diagnosticados no mesmo ambiente.

A raridade do evento TB-MDR na gravidez impôs a coleta de dados secundários retrospectivos através de anotações em prontuários, quando uma abordagem prospectiva seria mais adequada. Outra limitação foi a indisponibilidade de dados para a avaliar os fatores que contribuíram para a qualidade da terapia prévia ao esquema de multirresistência, como número de drogas, dosagens e duração dos tratamentos. Também, a ausência de informação sobre hospitalização no passado, reconhecida como potencial fonte de transmissão de cepas de tuberculose resistente, não permitiu verificar transmissão nosocomial.

Pode-se concluir que deve ser enfatizada a extrema importância da prevenção da resistência aos tuberculostáticos. O tratamento supervisionado é imperativo na TB-MDR pelos problemas relacionados à transmissibilidade, à adesão e às questões clínicas envolvidas. Nesta série de casos, o esquema terapêutico foi utilizado sem efeitos adversos para os $\mathrm{RN}$; porém, o planejamento familiar deve ser fortemente recomendado.

\section{CONFLITO DE INTERESSE}

Os autores declaram não haver nenhum tipo de conflito de interesse no desenvolvimento do estudo.

\section{REFERÊNCIAS}

1. Hamadeh MA, Glassroth J. Tuberculosis and pregnancy. Chest 1992; 101:1114-1120.

2. Nitta AT, Milligan D. Management of four pregnant women with multidrugresistant tuberculosis. Clin Infect Dis 1999; 28:1298-1304.

3. Shin S, Guerra D, Rich M, Seung KJ, Mukherjee J, Joseph K, et al. Treatment of multidrug resistant tuberculosis during pregnancy: a report of 7 cases. Clin Infect Dis 2003; 36:996-1003.

4. Lessnau KD, Qarah S. Multidrug resistant tuberculosis in pregnancy: case report and review of the literature. Chest 2003; 123:953-956.

5. Takashima T, Danno K, Tamura Y, Nagai T, Matsumoto T, Han Y, et al. Treatment outcome of patients with multidrug resistant pulmonary tuberculosis during pregnancy. Kekkaku 2006; 81:413-418.

6. Khan M, Pillay T, Moodley J, Ramjee A, Padayatchi N. Pregnancies complicated by multidrug resistant tuberculosis and HIV co-infection in Durban, South Africa. Int J Tuberc Lung Dis 2007; 11:706-708.

7. Tabarsi P, Baghaei P, Mirsaeidi M, Amiri M, Mansouri D, Novin A, et al. Multidrug resistant tuberculosis in pregnancy: need for more intensive treatment. Infection 2007; 35:477-478.
8. Palacios E, Dallman R, Muñoz M, Hurtado R, Chalco K, Guerra D, et al. Drug resistant tuberculosis and pregnancy: treatment outcomes of 38 cases in Lima, Peru. Clin Infect Dis 2009; 48:1413-1419.

9. Tabarsi P, Moradi A, Baghaei P, Marjani M, Shamaei M, Mansouri N, et al. Standardised second line treatment of multidrug resistant tuberculosis during pregnancy. Int J Tuberc Lung Dis 2011; 15:547-550.

10. Oliveira HB, Moreira Filho D. Abandono de tratamento e recidiva da tuberculose: aspectos de episódios prévios, Campinas, SP, Brasil, 1993-1994. Rev Saude Publica 2000; 34:437-443.

11. Holtz TH, Sternberg M, Kammerer S, Laserson KF, Riekstina V, Zarovska E, et al. Time to sputum culture conversion in multidrug resistant tuberculosis: predictors and relationship to treatment outcome. Ann Intern Med 2006; 144:650-659.

12. Furin JJ, Mitnick CD, Shin SS, Bayona J, Becerra MC, Singler JM, et al. Occurrence of serious adverse effects in patients receiving community-based therapy for multidrug-resistant tuberculosis. Int J Tuberc Lung Dis 2001; 5:648-655.

13. Teixeira L, Perkins MD, Johnson JL, Keller R, Palaci M, Valle-Dettoni V, et al. Infection and disease among household contacts of patients with multidrug resistant tuberculosis. Int J Tuberc Lung Dis 2001; 5:321-328.

14. SchaafHS, Gie RP, Kennedy M, Beyers N, Hesseling PB, Donald PR. Evaluation of young children in contact with adult multidrug-resistant pulmonary tuberculosis: a 30-month follow-up. Pediatrics 2002; 109:765-771.

15. Ormerod P. Tuberculosis in pregnancy and the puerperium. Thorax $2001 ; 56: 494-499$ 